\title{
The Interaction Effect of Mixing Starter Cultures on Homemade Natural Yogurt's pH and Viscosity
}

\author{
Hadi A. DAhlan ${ }^{*}$ AND Norrakiah A. SANi ${ }^{\mathrm{a}}$ \\ ${ }^{a}$ School of Chemical Sciences and Food Technology, Faculty of Science and Technology, Universiti Kebangsaan \\ Malaysia, 43600 UKM Bangi, Selangor Malaysia \\ ${ }^{*}$ Corresponding author \\ hadiakbar1591@rocketmail.com \\ TEL: $+6016-3274781$ \\ FAX: $+603-89213232$ \\ Received: 31 August 2016; Published online: 18 October 2017 \\ Invited paper from the IFA-event "Food Rheology and Texture" e-conference for undergraduate MASTER \\ students
}

\begin{abstract}
Dairy yogurts are common food products consumed by people all over the world. Due to the simple process, many people have made their own natural yogurt at home. The fermentation due to the starter culture causes the textural properties of dairy yogurt. However, the literature is surprisingly scarce on the topic of starter culture interactions in the development of textural properties of dairy yogurt. This study investigated the interaction effect of three common starter cultures, Lactobacillus acidophilus, Lactobacillus bulgaricus and Streptococcus thermophiles, on the viscosity of homemade yogurt. Using Design Expert software, a 10-run mixture model experiment was designed to examine the textural properties developed by single or multiple inoculation of these starter cultures. All yogurt formulations reached the isoelectric point of milk and had pHs in the range 3.97 to 4.32. Yogurt formulations with L. acidophilus and $S$. thermophilus resulted in viscosities which were similar to commercial yogurt viscosity (1.77 Pa.s), while L. bulgaricus resulted in yogurt with a lower viscosity. Based on the mixture model, L. acidophilus had most influence on the yogurt viscosity, followed by $S$. thermophilus and $L$. bulgaricus. In conclusion, L. acidophilus can be used as a single starter culture or combined with other starter cultures to develop high viscosity homemade yogurt. A Combination of $S$. thermophilus and L. acidphilus can also be used to develop high viscosity yogurts. However, L. bulgaricus should not be inoculated alone or become a dominant ratio in multiple starter culture inoculation as it will decrease the overall homemade yogurt viscosity.
\end{abstract}

Keywords: Viscosity; Yogurts; Starter culture; Lactobacillus acidophilus; Lactobacillus bulgaricus; Steptococcus thermophilus

\section{Introduction}

Yogurt is one of the basic fermented foods consumed worldwide. It is produced by fermenting bacteria in milk at appropriate temperatures (incubating temperature) and time. Yogurt's development is said to originate from the nomads in the Middle East (Tamime \& Robin- son, 1999). The fermenting bacteria are now marketed as starter culture products (Mazzoli, Bosco, Mizrahi, Bayer, \& Pessione, 2014). Each starter culture will coagulate the substrate where it is inoculated. The coagulation is due to accumulation of lactic acid that converts casein into lumps of micelles (Lee \& Lucey, 2010). Lactobacillus acidophilus, Lactobacillus bulgari- 
cus and Steptococcus thermophilus are some of the most utilized starter cultures in the production of dairy products (Delorme, 2008). Both L. acidophilus and L. bulgaricus possess proteolytic capability, that is the capable of hydrolyzing food proteins to peptides and amino acids to contribute to texture, taste and aroma of fermented products (McSweeney \& Sousa, 2000). The key role of $S$. thermophilus is to breakdown lactose to lactic acid, and therefore reduce $\mathrm{pH}$ to preserve the product. S. thermophilus also produces secondary metabolites, such as exopolysaccharides and flavoured compounds (Broadbent, McMahon, Welker, Oberg, \& Moineau, 2003).

These probiotic starter cultures are now available for public use and sold in specialised shops for food ingredients. Thus, the general public can easily produce homemade natural yogurt for personal consumption (Fisberg \& Machado, 2015). However, homemade natural yogurts are usually made without preservatives, unlike most commercial yogurts (O'Rell \& Chandan, 2013; Smith, 2015). The literature regarding homemade natural yogurt is surprisingly scarce. Thus, this study aims to contribute towards the body of literature by investigating the interaction effect of mixing starter cultures on homemade natural yogurt's $\mathrm{pH}$ and viscosity.

\section{Materials and Methods}

\section{$2.1 \quad$ Yogurt Preparation}

Three common yogurt starter cultures were used: Lactobacillus acidophilus, Lactobacillus bulgaricus and Streptococcus thermophilus. All three starter cultures were obtained from Custom Probiotics (Custom Probiotics Inc. ${ }^{\mathrm{TM}}$, California) in powder form. Pasteurized milk fresh from a local dairy farm in Sabak Bernam, Selangor, under the Farm Fresh Milk brand was obtained. $100 \mathrm{ml}$ of the dairy milk was poured into respective cups, before it was inoculated with approximately $1 \mathrm{~g}$ of starter cultures. Table 1 shows the mixture design that was used to make the 10 yogurt formulations, where each yogurt contains a different ratio of the 3 selected probiotic strains. The inoculation volume was based on the weight of the single starter culture powder or cumulative of multiple starter culture powders. For example, formulation 7 required one third of all three starter cultures. Therefore, $0.33 \mathrm{~g}$ of all three starter cultures was inoculated into the dairy milk. After inoculation with starter cultures, the milk samples were homogenized using an orbital shaker (MS Major Science, MS-NOR30, USA) for 15 minutes at $100 \mathrm{rpm}$.

The mixture design was a simplex Centroid design, and it was developed on Design-Expert ${ }^{\circledR}$ Version 6.9 (State-Ease, Inc. Minneapolis, 2003). All homemade yogurts were incubated at $37{ }^{\circ} \mathrm{C}$ for 24 hours via a Trio Yogurt Maker (Trio Kaden (M) Sdn Bhd, TYM-7, Malaysia). All yogurt samples were analyzed within a maximum of 3 days to ensure freshness.

\subsection{Yogurt $\mathrm{pH}$ Measurement}

Yogurt $\mathrm{pH}$ measurements were made using a benchtop pH meter (Mettler Toledo Inc., Parkway, USA). The pH-meter was calibrated before it was used for yogurt measurement. The yogurt samples were measured immediately after the incubation period (Sadler \& Murphy, 2010).

\subsection{Yogurt Viscosity Measurement}

A rotational viscometer (Brookfield, model DV II, USA) was used to measure the viscosity of the dairy yogurts. Approximately $50 \mathrm{ml}$ of yogurt in a $50-\mathrm{ml}$ beaker was used as a sample during measurement. The viscosity measurements were made at room temperature $\left(27^{\mathrm{O}} \mathrm{C}\right)$ using a Brookfield LV spindle no. 4 at 10 r.p.m..

\section{Results and Discussion}

\section{$3.1 \mathrm{pH}$ of Yogurt}

Table 2 shows the $\mathrm{pH}$ values of the 10 yogurt formulations which lie within the range 3.97 to 4.32. This $\mathrm{pH}$ range is consistent with the 4.0 to $4.5 \mathrm{pH}$ values reported by Gahruie, Eskandari, Mesbahi, and Hanifpour (2015) for commercial yogurts.

Single inoculation $L$. bulgaricus produced the 
Table 1: Mixture experiment design of multiple species inoculation

\begin{tabular}{cccc}
\hline \multicolumn{5}{c}{ Sample: Dairy milk Inoculation volume : $\mathbf{~ g}$} \\
Run & Lactobacillus & acidophilus & Lactobacillus \\
bulgaricus & Streptococcus thermophilus \\
\hline 1 & 1 & 0 & 0 \\
2 & 0 & 1 & 0 \\
3 & 0 & 0 & 1 \\
4 & $\frac{1}{2}$ & 0 & $\frac{1}{2}$ \\
5 & $\frac{1}{2}$ & 1 & $1 / 3$ \\
6 & $\frac{1}{2}$ & $1 / 3$ & $1 / 6$ \\
7 & 0 & $1 / 6$ & $1 / 6$ \\
8 & $1 / 3$ & $2 / 3$ & $2 / 3$ \\
9 & $2 / 3$ & $1 / 6$ & \\
10 & $1 / 6$ & &
\end{tabular}

lowest yogurt $\mathrm{pH}$ of the ten formulations (Table 2 ). Dual starter inoculation containing $L$. bulgaricus (formulations 4 and 6 ) also had low $\mathrm{pH}$ values when compared to the other yogurt formulations. This is probably one of the reasons why the dairy industry utilises multiple inoculations when producing commercial yogurt (Leroy \& De Vuyst, 2004).

\subsection{Viscosity of Yogurt}

Table 3 shows the viscosity of the 10 yogurt formulations which were within the range 2.307 $\mathrm{Pa} \cdot \mathrm{s}$ to $1.058 \mathrm{~Pa} \cdot \mathrm{s}$. According to Lee and Lucey (2010), gelation occurs when pH is just above the dairy milk isoelectric point ( $\mathrm{pH} \mathrm{5.2)}$ and the observed viscosities are indicative of gelation in all 10 formulations. Normally, commercial yogurt contains multiple starter cultures and has an average viscosity of $1.77 \mathrm{~Pa} \cdot \mathrm{s}$ (Ares et al., 2007). However, not all commercial yogurts declare which starter cultures are used. Several yogurt formulations are near or exceed the commercial yogurt viscosity (formulations 3, 5, 6 and 10) (Table 3).

Yogurt formulations with $L$. acidophilus have the highest viscosity (formulations 1 and 8), followed by those with $S$. thermophilus. When $L$. acidophilus and $S$. thermophilus are combined (formulation 5), the yogurt viscosity increases above that of $S$. thermophilus alone. When L. acidophilus and L. bulgaricus were combined (for- mulation 4), the resulting yogurt had a higher viscosity than the yogurt with a single $L$. bulgaricus inoculation.

L. acidophilus seems to have high viscosity capability. This was shown even in multiple starter culture yogurt formulations, for example, in formulation 8 where L. acidophilus was the dominant starter culture ratio. Conversely, L. bulgaricus seems to decrease yogurt viscosity whenever it is used to inoculate. Yogurt formulations with dominant $L$. bulgaricus starter culture ratio have lower viscosities than yogurt formulations without L. bulgaricus inoculations (formulation 6 and 9).

\subsection{Model System}

Table 4 shows coefficient estimates, regression coefficient $\left(\mathrm{R}^{2}\right)$, model significance and lack of fit results for dairy yogurt viscosity. The values indicate the significance of equations or models that were developed from the design. If the probability of the $\mathrm{F}$ value obtained is less than 0.05 , it means that model or equation can be developed confidently $(\mathrm{p}<0.05)$. According to Henika (1982), $\mathrm{R}^{2}$ values of more than 0.75 can be used for prediction purposes. However, the closer the $\mathrm{R}^{2}$ is to unity, the better the empirical model fits the actual data. The lack-of-fit test is a measure of the failure of the model to represent the data in the experimental domain at which points were not included in the regression, or in other words, 
Viscosity of homemade natural yogurt | 155

Table 2: pH of ten dairy yogurt formulations

\begin{tabular}{rcccc}
\hline \multirow{2}{*}{ Formulation } & \multicolumn{3}{c}{ Ratio Strain } & pH \\
\cline { 2 - 5 } & $\begin{array}{c}\text { Lactobacillus } \\
\text { acidophilus }\end{array}$ & $\begin{array}{c}\text { Lactobacillus } \\
\text { bulgaricus }\end{array}$ & $\begin{array}{c}\text { Streptococcus } \\
\text { thermophilus }\end{array}$ & $\begin{array}{c}\text { Natural } \\
\text { Yogurt }\end{array}$ \\
\hline 1 & 1.00 & - & - & $4.31 \pm 0.02^{a}$ \\
2 & - & 1.00 & - & $3.97 \pm 0.03^{d}$ \\
3 & - & - & 1.00 & $4.24 \pm 0.01^{b}$ \\
4 & 0.50 & 0.50 & - & $4.04 \pm 0.03^{c}$ \\
5 & 0.50 & - & 0.50 & $4.29 \pm 0.01^{a b}$ \\
6 & - & 0.50 & 0.50 & $4.09 \pm 0.01^{c}$ \\
7 & 0.33 & 0.33 & 0.33 & $4.31 \pm 0.03^{a}$ \\
8 & 0.67 & 0.16 & 0.16 & $4.30 \pm 0.02^{a b}$ \\
9 & 0.16 & 0.67 & 0.16 & $4.31 \pm 0.03^{a}$ \\
10 & 0.16 & 0.16 & 0.67 & $4.32 \pm 0.02^{a}$ \\
\hline
\end{tabular}

a-e Different letters indicate significant difference $(\mathrm{p} \leq 0.05)$

Table 3: Viscosity of ten dairy yogurt formulations

\begin{tabular}{rcccc}
\hline Formulation & \multicolumn{3}{c}{ Ratio Strain } & Viscosity (Pa·s) \\
\cline { 2 - 5 } & $\begin{array}{c}\text { Lactobacillus } \\
\text { acidophilus }\end{array}$ & $\begin{array}{c}\text { Lactobacillus } \\
\text { bulgaricus }\end{array}$ & $\begin{array}{c}\text { Streptococcus } \\
\text { thermophilus }\end{array}$ & $\begin{array}{c}\text { Dairy } \\
\text { Yogurt }\end{array}$ \\
\hline 1 & 1.00 & - & - & $2.1597 \pm 0.31^{a}$ \\
2 & - & 1.00 & - & $1.2410 \pm 0.00^{d e}$ \\
3 & - & - & 1.00 & $1.7660 \pm 0.07^{a b c d}$ \\
4 & 0.50 & 0.50 & - & $1.9250 \pm 0.05^{a b c}$ \\
5 & 0.50 & - & 0.50 & $1.8970 \pm 0.17^{a b c}$ \\
6 & - & 0.50 & 0.50 & $1.5940 \pm 0.15^{b c d e}$ \\
7 & 0.33 & 0.33 & 0.33 & $1.5050 \pm 0.08^{c d e}$ \\
8 & 0.67 & 0.16 & 0.16 & $2.3077 \pm 0.21^{a}$ \\
9 & 0.16 & 0.67 & 0.16 & $1.0579 \pm 0.14^{e}$ \\
10 & 0.16 & 0.16 & 0.67 & $1.6840 \pm 0.28^{b c d}$ \\
\hline
\end{tabular}

a-e Different letters indicate significant difference $(\mathrm{p} \leq 0.05)$ 
variations in the models cannot be accounted for by random error.

The F-value obtained was less than 0.05 (0.0109). The $\mathrm{R}^{2}$ for yogurt was 0.6334 . The yogurts also displayed statistically insignificant lack-offit, thus indicating that the experimental data was satisfactorily accepted for model fitting even though the $\mathrm{R}^{2}$ of the dairy yogurt is less than 0.75 , it can be used with confidence (Henika, 1982; Yusnita, Aida, Maskat, \& Aminah, 2007). According to the Design Expert software, there were interactions between binary and tertiary components. However, these interactions were not significant and were removed in order to develop a more robust model using the significant single components.

The canonical Scheffe's equations for the dairy yogurt viscosities were obtained from the software. The models were analyzed as follows. The positive $(+)$ sign in the equation meant that the response values increased with an increase of the variables, whether it is linear $(\mathrm{A}, \mathrm{B}, \mathrm{C})$, binary $(\mathrm{AB}, \mathrm{AC}, \mathrm{BC})$ or ternary combination $(\mathrm{AC}(\mathrm{A}-$ $\mathrm{C})$ ). There is an inverse meaning when a negative (-) sign is shown on the variables.

The canonical Scheffe's equation for dairy yogurt viscosity is shown in equation 1 :

Viscosity $=0.222 \cdot A+0.12236 \cdot B+0.15282 \cdot C$

where A is L. acidophilus, B is L. bulgaricus and $\mathrm{C}$ is S. thermophilus.

In equation 1, the single inoculation of $L$. acidophilus had the highest influence on the homemade dairy yogurt viscosity followed by $S$. thermophilus and L. bulgaricus. L. acidohpilus has the highest mathematical weigh $(+0.222)$. This is because L. acidophilus has the ability to convert $23 \%$ of lactose into lactic acid. The accumulating lactic acid then contributes to the viscosity of the yogurt (Anjum et al., 2014). Formulations 1 and 8 of the homemade dairy yogurts validate this statement.

The second highest was $S$. thermophilus $(+0.15282)$. S. thermophilus has the ability to breakdown lactose into glucose and galactose. $S$. thermophilus then proceeds to digest glucose monomers for growth (Broadbent et al., 2003; Drouault, Anba, \& Corthier, 2002). Almost all formulations that contain $S$. thermophilus had high viscosity, especially when paired with L. acidophilus (formulation 8 and 10). This is because $L$. acidophilus also benefited from the breakdown of lactose, since it utilizes galactose in its growth (Noh \& Gilliland, 1993).

$L$. bulgaricus had the lowest influence in dairy yogurt viscosity $(+0.12236)$. Although $L$. bulgaricus was able to breakdown peptides into free amino acids that can contribute to viscosity and sensory quality (McSweeney \& Sousa, 2000), it relies on other starter cultures for its growth. $L$. bulgaricus requires galactose or glucose for its metabolism but it cannot breakdown the lactose in order to feed itself (Nguyen et al., 2012; Zourari, Accolas, \& Desmazeaud, 1992). This is an example of why dairy yogurt requires an emulsifier in its formulation (O'Rell \& Chandan, 2013; Smith, 2015).

Table 4: Coefficient estimates, model significance, regression coefficient and lack-of-fit values for Dairy yogurt viscosity

\begin{tabular}{cc}
\hline & Ratio Strain in Yogurt \\
\cline { 2 - 2 } Coefficients & Dairy Yogurt \\
\hline A & +0.22222 \\
B & +0.12236 \\
C & +0.15282 \\
Model (Prob> F) & 0.0109 \\
Regression Coefficient $\left(\mathrm{R}^{2}\right)$ & 0.6334 \\
Lack of fit & 0.7218 \\
\hline
\end{tabular}

\section{Conclusions}

There are significant differences in the texture of homemade dairy yogurts inoculated with different ratios of starter culture. All homemade yogurt formulations reached the isoelectric point of milk and had pHs in the range 3.97 to 4.32 . The most viscous homemade yogurt $(2.3 \mathrm{~Pa} \cdot \mathrm{s})$ came from a multiple inoculation formulation with $L$. acidophilus as the dominant ratio. When results are fitted into a Scheffe's canonical special quadratic equation for three components, the model shows that $L$. acidophilus brings the most positive influence to increase homemade yogurt viscosity. This is followed by $S$. thermophilus and L. bulgaricus. In conclusion, L. acidophilus can be used as a single starter culture or combined 
with other starter cultures to develop high viscosity homemade natural yogurts. A combination of S. thermophilus and L. acidophilus can also be used to develop high viscosity homemade yogurts. However, L. bulgaricus should not be inoculated alone or become the dominant ratio in a multiple starter culture inoculation since it will decrease the overall viscosity of homemade yogurt.

\section{Acknowledgements}

This research was supported by DIP-2014-007 from the Universiti Kebangsaan Malaysia. We would like to express our gratitude for support with the research funding.

\section{References}

Anjum, N., Maqsood, S., Masud, T., Ahmad, A., Sohail, A., \& Momin, A. (2014). Lactobacillus acidophilus: characterization of the species and application in food production. Critical Reviews in Food Science and Nutrition, 54(9), 1241-1251. doi:10.1080/ 10408398.2011.621169

Ares, G., Gonçalvez, D., Pérez, C., Reolón, G., Segura, N., Lema, P., \& Gámbaro, A. (2007). Influence of gelatin and starch on the instrumental and sensory texture of stirred yogurt. International Journal of Dairy Technology, 60(4), 263-269. doi:10. 1111/j.1471-0307.2007.00346.x

Broadbent, J. R., McMahon, D. J., Welker, D. L., Oberg, C. J., \& Moineau, S. (2003). Biochemistry, genetics, and applications of exopolysaccharide production in Streptococcus thermophilus: a review. Journal of Dairy Science, 86(2), 407-423. doi:10. 3168/jds.S0022-0302(03)73619-4

Delorme, C. (2008). Safety assessment of dairy microorganisms: Streptococcus thermophilus. International Journal of Food Microbiology, 126(3, SI), 274-277. doi:10. 1016/j.ijfoodmicro.2007.08.014

Drouault, S., Anba, J., \& Corthier, G. (2002). Streptococcus thermophilus is able to produce a $\beta$-galactosidase active during its transit in the digestive tract of germ-free mice. Applied and Environmental Microbiology, 68(2), 938-941. doi:10.1128/AEM. 68.2.938-941.2002

Fisberg, M. \& Machado, R. (2015). History of yogurt and current patterns of consumption. Nutrition Reviews, 73(1), 4-7. doi:10. 1093/nutrit/nuv020

Gahruie, H. H., Eskandari, M. H., Mesbahi, G. \& Hanifpour, M. A. (2015). Scientific and technical aspects of yogurt fortification: a review. Food Science and Human Wellness, 4 (1), 1-8. doi:10.1016/j.fshw.2015.03.002

Henika, R. G. (1982). Use of response-surface methodology in sensory evaluation. Food Technology, 36(11), 96-101.

Lee, W. J. \& Lucey, J. A. (2010). Formation and physical properties of yogurt. Asianaustralasian Journal of Animal Sciences, $23(9), 1127-1136$.

Leroy, F. \& De Vuyst, L. (2004). Lactic acid bacteria as functional starter cultures for the food fermentation industry. Trends in Food Science \& Technology, 15(2), 67-78. doi:10.1016/j.tifs.2003.09.004

Mazzoli, R., Bosco, F., Mizrahi, I., Bayer, E. A., \& Pessione, E. (2014). Towards lactic acid bacteria-based biorefineries. Biotechnology Advances, 32(7), 1216-1236. doi:10.1016/ j.biotechadv.2014.07.005

McSweeney, P. L. H. \& Sousa, M. J. (2000). Biochemical pathways for the production of flavour compounds in cheeses during ripening: a review. Lait, 80(3), 293-324.

Nguyen, T.-T., Nguyen, H. A., Arreola, S. L., Mlynek, G., Djinović-Carugo, K., Mathiesen, G., ... Haltrich, D. (2012). Homodimeric $\beta$-galactosidase from Lactobacillus delbrueckii subsp. bulgaricus dsm 20081: expression in Lactobacillus plantarum and biochemical characterization. Journal of Agricultural and Food Chemistry, 60(7), 1713-1721. PMID: 22283494. doi:10.1021/ jf203909e

Noh, D. \& Gilliland, S. (1993). Influence of bile on cellular integrity and $\beta$-galactosidase activity of Lactobacillus acidophilus. Journal of Dairy Science, 76 (5), 1253-1259. doi:10. 3168/jds.S0022-0302(93)77454-8

O'Rell, K. \& Chandan, R. C. (2013). Manufacture of various types of yogurt. In 
Manufacturing yogurt and fermented milks (pp. 263-295). John Wiley \& Sons. doi:10. 1002/9781118481301.ch12

Sadler, G. D. \& Murphy, P. A. (2010). Ph and titratable acidity. In Food analysis (pp. 219-238). Boston, MA: Springer US. doi:10.1007/978-1-4419-1478-1_13

Smith, D. K. (2015). Yogurt made simple. Pullman, Washington: Washington State University. Extension.

Tamime, A. Y. \& Robinson, R. K. (1999). Yoghurt: science and technology. (Chap. Introduction, pp. 1-5). Woodhead Publishing.

Yusnita, H., Aida, W. M. W., Maskat, M. Y., \& Aminah, A. (2007). Processing performance of coated chicken wings as affected by wheat, rice and sago flours using response surface methodology. International Journal of Food Science and Technology, 42(5), 535-542. doi:10.1111/j.1365-2621. 2006.01262.x

Zourari, A., Accolas, J. P., \& Desmazeaud, M. J. (1992). Metabolism and biochemical characteristics of yogurt bacteria - a review. Lait, 72(1), 1-34. 